\title{
Hyperspectral Image Denoising via Minimizing the Partial Sum of Singular Values and Superpixel Segmentation
}

\author{
Yang Liu ${ }^{\mathrm{a}}$, Caifeng Shan ${ }^{\mathrm{b}}$, Quanxue Gao ${ }^{\mathrm{a}}$, Xinbo Gao ${ }^{\mathrm{a}}$, Jungong $\mathrm{Han}^{\mathrm{c}}$, Rongmei Cui ${ }^{\mathrm{a}}$ \\ ${ }^{a}$ State Key Laboratory of Integrated Services Networks, Xidian University, Xi'an, China. \\ ${ }^{b}$ the Medical Image Analysis, Philips Research, High Tech Campus 34, Eindhoven 5656AE, NL. \\ ${ }^{c}$ the School of Computing and Communications at Lancaster University, Lancaster, UK.
}

\begin{abstract}
Hyperspectral images (HSIs) are often corrupted by noise during the acquisition process, thus degrading the HSI's discriminative capability significantly. Therefore, HSI denoising becomes an essential preprocess step before application. This paper proposes a new HSI denoising approach connecting Partial Sum of Singular Values (PSSV) and superpixel segmentation named as SS-PSSV, which can remove the noise effectively. Based on the fact that there is a correlation between different bands of the same signal, it is easy to know the property of low rank. To this end, PSSV is utilized, and in order to better tap the low-rank attribute of samples, we introduce the superpixel segmentation method, which allows samples of the same type to be grouped in the same sub-block as much as possible. Extensive experiments display that the proposed algorithm outperforms the state-of-the-art.

Keywords: PSSV, superpixel segmentation, hyperspectral images, denoising
\end{abstract}

\section{Introduction}

In the recent years, hyperspectral images (HSIs) become more and more popular, which are being used in a wide range of fields, such as agriculture [1], terrain classification [2], geological analysis [3], and military surveillance [4, 5]. However,

5 hyperspectral images often suffer from noises in the process of data acquisition, due to the effects of photon, the sensor, and calibration error. Therefore, HSI denoising is an 
important processing setup and significantly affects on the performance of subsequent applications.

Recently, many HSIs' denoising approaches have been proposed such as $[6,7,8,9]$. As known, low-rank approximation as a powerful method is becoming more and more popular in image analysis field, computer vision and web search [10,11], or in the denoising problem of exploring and searching low-dimensional structure from highdimensional data in the past years. The aim of low-rank matrix approximation-based image recovery method is to remove the sparse noise due to the prior knowledge that some components from the clean image are regarded as low-rank. With aid of the difference between signal and noise, noise can be removed efficiently in the wavelet domain. And meanwhile, low-rank matrix approximation methods, such as Principal Component Analysis (PCA) [12] and matrix factorization [13, 14] are widely used to find the best approximation of an underlying low-rank structure of data.

In the conventional PCA [12], the goodness-of-fit of data is evaluated by $L_{2}$-norm, which is very sensitive to outliers. To address this problem and to recover the lowrank matrix while rejecting outliers, Robust PCA [11] improves PCA by not using $L_{2}$ norm, so a rank minimization has been proposed and gained much interests in computer vision $[15,16,17]$. Early works in RPCA tried to reduce the effects of outliers by random sampling [18] or robust M-estimator $[19,20]$ to identify outliers or penalize data with large errors. However, these methods share some limitations: either they are sensitive to the choice of parameters, or they are not scalable enough in running time. To further improve the above algorithms, PSSV [11] incorporated a prior information about the target rank, which minimizes the partial sum of singular values to encourage the target rank constraint. In view of its great advance, PSSV [11] is also exploited for hyperspectral image denoising in this paper.

Motivated by the fact that PSSV [11] is successful in dynamic object, we want to test whether it is suitable for hyperspectral images. Therefore, PSSV [11] is introduced into our model, and we notice a fact that there is a high correlation between different modalities from the same signal although the small difference exists, thus implying a low rank property should be an appropriate prior knowledge. In order to make further use of low-rank attributes, we decided to adopt superpixels to segment the image, which 
help group the same type of samples into the same sub-block as much as possible.

This paper is organized as follows: Section 2 overviews the related work and Section 3 presents the process of hyperspectral denoising, followed by the experiments in Section 4. In addition, Section 5 concludes this paper and discusses future work.

\section{Related Works}

In the past years, many methods have been adopted to reduce noise in HSI band by band or pixel or pixel [21]. However, these denoising results are not satisfactory, because the relationship between the spatial and spectral bands is not premeditated simultaneously, that is to say, only the noise in spatial or spectral region is removed.

Low rank representation (LRR) has been used in HSI analysis [22]. Lu et al [23] introduced LRR to remove stripe noise in HSI based on correlation among different bands, and a graph regularization is considered for the local geometrical structure. Zhang [24] proposed a HSI denoising method based on low rank matrix recovery(LRMR), i.e.

$$
\min _{\mathbf{A}, \mathbf{E}}\|\mathbf{A}\|_{*}+\lambda\|\mathbf{E}\|_{1} \text { s.t. }\|\mathbf{O}-\mathbf{A}-\mathbf{E}\|_{F} \leq \delta
$$

where $\mathbf{O}$ is the input, and aim of the model is to recover clean matrix $\mathbf{A}$ from $\mathbf{O}$. In [24], LRMR achieves perfect performance while the uncorrupted HSIs comply with the low rank assumption. However, LRMR only considers that the rank is low, but does not limit the extent of the low rank.

PSSV [25] extends the extent of the low rank, and the rank is accurate to a specific number. As in [25], PSSV is proven to be effective in dynamic object. In this paper, we will introduce PSSV into hyperspectral field, and latter experiment results show its effectiveness. Furthermore, we also introduce the superpixel segmentation into our model, which further improves the denoising effect.

\section{Hyperspectral Image Denoising}

\section{1. superpixel segmentation}

A superpixel is actually a cluster of pixels having the same type, so it can transform a pixel-level map into a district-level map. 


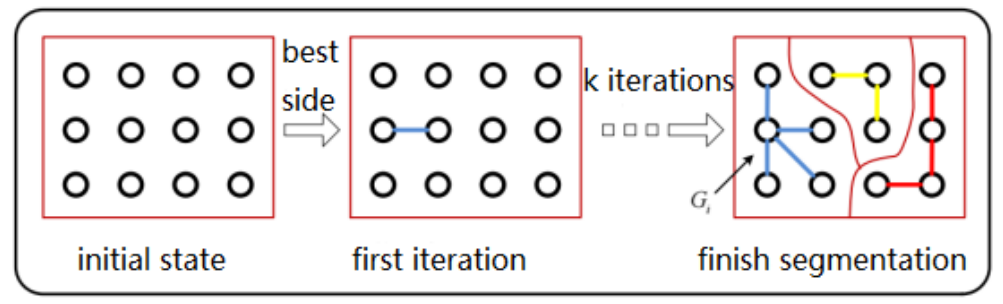

Figure 1: super pixel segmentation process.

In this paper, superpixel segmentation based on entropy rate is utilized and the objective function is,

$$
\max -\sum_{i} u_{i} \sum_{j} p_{i, j}(A) \log \left(p_{i, j}(A)\right)+\lambda\left(-\sum_{i} p z_{A}(i) \log \left(p z_{A}(i)\right)-N_{A}\right)
$$

where $\lambda$ is to balance the weight between the two terms, $N_{A}$ is the number of connected components and $u\left(u_{1}, u_{2}, \ldots u_{i}, \ldots\right)$ satisfys a smooth distribution and where $p z_{A}(i)=$ $\frac{\left|S_{i}\right|}{|V|}, i=1,2, \ldots, N_{A}$. Here, $S_{i}$ is the i-th super pixel block, $V$ represents a collection of all pixels, and $\|$ indicates the number of pixels in the image block. Besides, in which

$$
p_{i, j}(A)= \begin{cases}\frac{\omega_{i, j}}{\omega_{i}} & \text { if } i \neq j, \text { and } e_{i, j} \in A \\ 0 & \text { if } i \neq j, \text { and } e_{i, j} \notin A \\ 1-\frac{\sum_{j: e_{i, j} \in A} \omega_{i, j}}{\omega_{i}} & \text { if } i=j\end{cases}
$$

where $e_{i, j}$ is an edge that connects the $\mathrm{i}$-th and $\mathrm{j}$-th pixels, and $A$ is the set of edges. Here, $\omega_{i, j}=\exp \left(-\frac{d\left(v_{1}, v_{2}\right)^{2}}{2 \sigma^{2}}\right)$, and where $v_{1}, v_{2}$ are adjacent $\mathrm{i}$-th pixel and $\mathrm{j}$-th pixel, $d\left(v_{1}, v_{2}\right)$ dispalys the distance between adjacent $\mathrm{i}$-th pixel and $\mathrm{j}$-th pixel, $\sigma$ is a parameter set by person and $\omega_{i, j}$ is the similarity between $\mathrm{i}$-th pixel and $\mathrm{j}$-th pixel.

In this objective function, the first item ensures pixels with high similarity are grouped into the same superpixel and the second item favors clusters with same size. Therefore, this objective function can ensure to get both compact, homogeneous, and balanced clusters. And the rough process is shown in Figure 1.

\subsection{PSSV model}

For each divided sub-block, it mainly contains the same type of samples, and there is a high correlation between different bands in the same signal. So we consider to 
adopt the PSSV [25] model to explore low rank attribute in this paper and it is the updated version of RPCA [10]. As we know, the RPCA model was first proposed by Ma et al. [10] and the objective is to recover clean matrix $\mathbf{A}$. The formulation of this model is the following:

$$
\min _{\mathbf{A}, \mathbf{E}} \operatorname{rank}(\mathbf{A})+\lambda\|\mathbf{E}\|_{0} \quad \text { s.t. } \mathbf{O}=\mathbf{A}+\mathbf{E}
$$

where $\lambda$ is the regularization parameter. As can be seen, it is a nonconvex optimization problem, and there is no effective solution. Generally, the problem is usually converted into a tractable optimation problem by substituting the $l_{0}$-norm for the $l_{1}$-norm and the rank for the nuclear norm, so the following optimization problem is acquired:

$$
\min _{\mathbf{A}, \mathbf{E}}\|\mathbf{A}\|_{*}+\lambda\|\mathbf{E}\|_{1} \quad \text { s.t. } \mathbf{O}=\mathbf{A}+\mathbf{E}
$$

where $\|\bullet\|_{*}$ is the nuclear norm(i.e., the sum of the singular values). Regarding the solution for Eq. (5), various algorithms have been proposed, where Alternating Direction Method of Multiplier (ADMM) has shown to be quite effective. Apart from the standard nuclear norm relaxation, there also exist some works that study variants of nuclear norm to improve the performance of rank minimization. Among them, instead of minimizing nuclear norm, PSSV [25] considers to minimize partial sum of singular values. And it can be proven effective in dynamic object, but it is rarely used in the field of hyperspectral, therefore, we decide to adopt it on hyperspectral images. And the latter part of experiments also confirms its hyperspectral validity, in the following, and PSSV will be introduced briefly.

At first, the idea of PSSV [25] is as:

$$
\arg \min _{\mathbf{A}, \mathbf{E}}|\operatorname{rank}(\mathbf{A})-\mathbf{N}|+\lambda\|\mathbf{E}\|_{0} \text { s.t. } \mathbf{O}=\mathbf{A}+\mathbf{E}
$$

The aim of PSSV [25] is to recover a low-rank matrix A with closing to the rank N and a sparse matrix E. Unfortunately, the Eq. (6) is a NP-hard problem, so in order to deal with the case, the idea of [10] is utilized to relax it with an alternative tractable representation. And meanwhile, $\operatorname{rank}(\mathbf{A})-N$ is also relaxed with a projection operator. 
As a result, the first term of Eq. (6) is replaced with $\|\mathbf{A}\|_{p=N}$ i.e.

$$
\begin{aligned}
|\operatorname{rank}(\mathbf{A})-\mathbf{N}| & \approx\left|\|\mathbf{A}\|_{*}-\left\|\mathbf{P}_{\mathbf{N}}(\mathbf{A})\right\|_{*}\right| \\
& =\left|\sum_{i=1}^{\min (n, m)} \sigma_{i}(\mathbf{A})-\sum_{i=1}^{\mathbf{N}} \sigma_{i}(\mathbf{A})\right| \\
& =\sum_{i=\mathbf{N}+1}^{\min (n, m)} \sigma_{i}(\mathbf{A})=\|\mathbf{A}\|_{p=N}
\end{aligned}
$$

As mentioned above, the objective function of PSSV is gained in the following:

$$
\arg \min _{\mathbf{A}, \mathbf{E}}\|\mathbf{A}\|_{P=N}+\lambda\|\mathbf{E}\|_{1} \quad \text { s.t. } \mathbf{O}=\mathbf{A}+\mathbf{E}
$$

In addition, the solution to this problem will be discussed in the following.

Compared with standard nuclear norm, the advantage of PSSV is that it does not minimize the variance distribution of data with the help of target rank.

\subsection{Optimization}

For Eq. (8), it can be solved by ADMM proposed by Lin et al. [26]. With the help of this method, the objective function can be evolved into the following form:

$$
L_{u}(\mathbf{A}, \mathbf{E}, \mathbf{Z})=\|\mathbf{A}\|_{p=N}+\lambda\|\mathbf{E}\|_{1}+\langle\mathbf{Z}, \mathbf{O}-\mathbf{A}-\mathbf{E}\rangle+\frac{\mu}{2}\|\mathbf{O}-\mathbf{A}-\mathbf{E}\|_{F}^{2}
$$

where $\mathbf{Z} \in \mathbf{R}^{m \times n}$ is the Lagrange multiplier, and $\mu$ is a positive scalar. According to [26], Eq. (9) can be optimized by updating variable in turn while fixing the other variables invariant, so the problem above can be divided into two subproblems.

\subsubsection{Solving $\mathbf{A}^{*}$}

From the Eq. (9), we can obtain that

$$
\begin{aligned}
\mathbf{A}^{*} & =\arg \min _{\mathbf{A}} \mathbf{L}_{\mu k}\left(\mathbf{A}, \mathbf{E}_{k}, \mathbf{Z}_{k}\right) \\
& =\arg \min _{\mathbf{A}} \mu_{k}{ }^{-1}\|\mathbf{A}\|_{p=N}+\frac{1}{2}\left\|\mathbf{A}-\left(\mathbf{O}-\mathbf{E}_{k}+\mu_{k}{ }^{-1} \mathbf{Z}_{k}\right)\right\|_{F}^{2}
\end{aligned}
$$

For Eq. (10), the Partial Singular Value Thresholding (PSVT) operator [25] $\mathbf{P}_{N, \tau}[\bullet]$ can solve the problem. As in [25], we can obtain:

$$
\mathbf{A}_{k+1}=\mathbf{P}_{N, \mu_{k}^{-1}}\left(\mathbf{O}-\mathbf{E}_{k}+\mu_{k}^{-1} \mathbf{Z}_{k}\right)
$$


where

$$
\mathbf{P}_{N, \tau}[\mathbf{Y}]=\mathbf{U}_{\mathbf{Y}}\left(\mathbf{D}_{\mathbf{Y} 1}+\mathbf{S}_{\tau}\left[\mathbf{D}_{\mathbf{Y} 2}\right]\right) \mathbf{V}_{\mathbf{Y}}^{\mathrm{T}}=\mathbf{Y}_{1}+\mathbf{U}_{\mathbf{Y} 2} \mathbf{S}_{\tau}\left[\mathbf{D}_{\mathbf{Y} 2}\right] \mathbf{V}_{\mathbf{Y} 2}^{\mathrm{T}},
$$

and where $\mathbf{D}_{\mathbf{Y} 1}=\operatorname{diag}\left(\sigma_{1}, \ldots, \sigma_{N}, 0 \ldots 0\right), \mathbf{D}_{\mathbf{Y} 2}=\operatorname{diag}\left(0, \ldots, 0, \sigma_{N+1}, \ldots, \sigma_{l}\right)$, $\mathbf{S}_{\tau}[x]=\operatorname{sign}(x) \max (|x|-\tau, 0)$ is the soft-thresholding operator $[27,28]$. In addition, PSVT [29] enforces the target rank constraint through projection and it implicitly encourages the resulting matrix to meet the target rank.

\subsubsection{Solving $\mathbf{E}^{*}$}

From the Eq. (9), we can obtain that

$$
\begin{aligned}
\mathbf{E}^{*} & =\underset{\mathbf{E}}{\arg \min } L_{\mu k}\left(\mathbf{A}_{k+1}, \mathbf{E}, \mathbf{Z}_{k}\right) \\
& =\underset{\mathbf{E}}{\arg \min } \lambda \mu_{k}^{-1}\|\mathbf{E}\|_{1}+\frac{1}{2}\left\|\mathbf{E}-\left(\mathbf{O}-\mathbf{A}_{k+1}+\mu_{k}^{-1} \mathbf{Z}_{k}\right)\right\|_{F}^{2}
\end{aligned}
$$

As in [28], Eq. (13) can be solved as:

$$
\mathbf{E}_{k+1}=S_{\lambda \mu_{k}^{-1}}\left(\mathbf{O}-\mathbf{A}_{k+1}+\mu_{k}^{-1} \mathbf{Z}_{k}\right)
$$

where $S_{\tau}[x]=\operatorname{sign}(x) \max (|x|-\tau, 0)$ is the soft-thresholding operator [27, 28].

Finally, the whole solution process is summarized in the following:

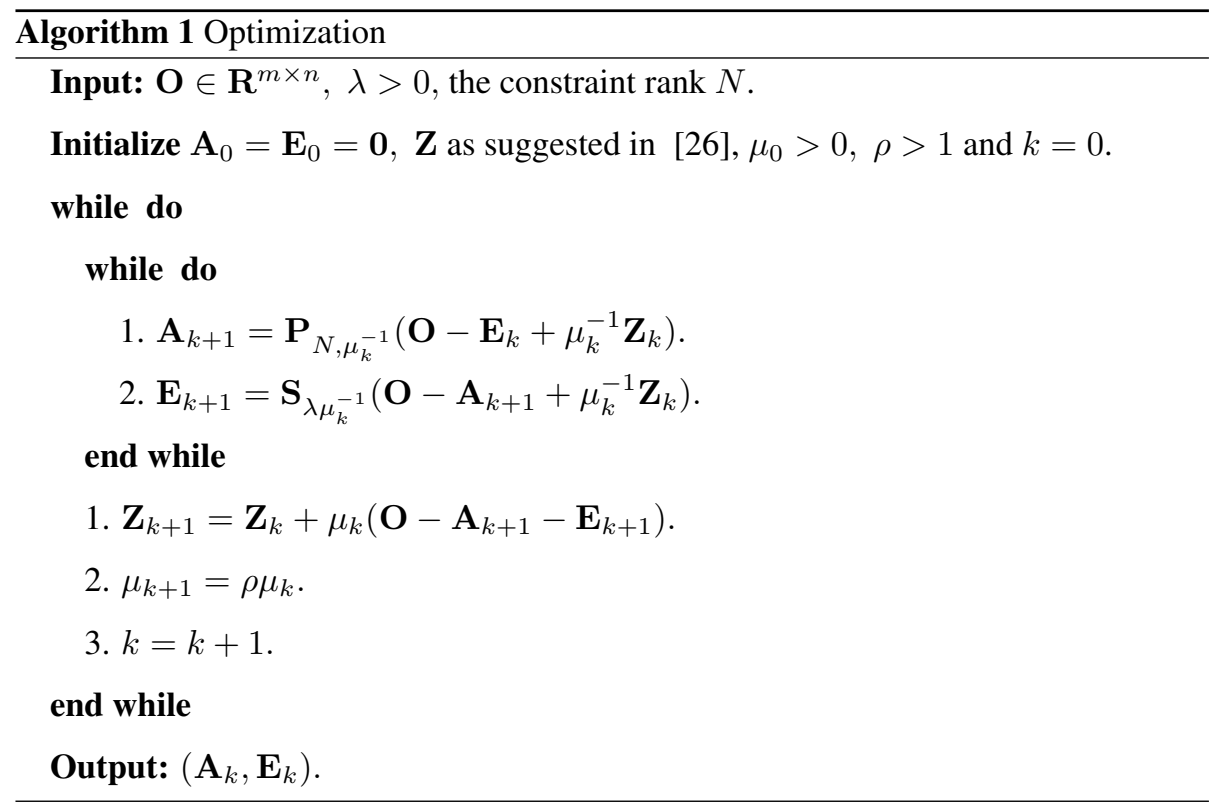




\section{Experiments}

To verify the effectiveness of our method, we carry out experiments on three hyperspectral data sets and make a comparison among the proposed algorithm with four algorithms including LRMR [24], robust Principal Component Analysis [10], robust Principal Component Analysis on Graphs [30], SS-LRR [31] and PSSV [25].

Among these contrast methods, LRMR [24] mainly adopts the low rank matrix recovery model and regards clean samples as low-rank, robust Principal Component Analysis [10] is an improvement for the PCA, and it adopts a rank minimization instead of $L_{2}$-norm, robust Principal Component Analysis on Graphs [30] incorporates spectral graph regularization into the Robust PCA framework, SS-LRR [31] combines superpixel segmentation and low-rank representation to denoise hyperspectral image and PSSV [25] is the update version of RPCA, minimizing partial sum of singular values, and it is firstly used in dynamic images, rarely in hyperspectral images. As we know, the several methods are state-of-the-art algorithms, so in order to verify the effectiveness of proposed method, we try to compare the proposed method with them.

In this paper, peak signal-to-noise ratio (PSNR) and structure similarity (SSIM) indices are used to give a quantitative assessment of the denoised results. For an HSI, we compute the value of two indices for images on different bands, and the mean value of these bands are calculated and denoted as Mean PSNR or Mean SSIM. Generally speaking, higher PSNR and higher SSIM values lead to a better denoised result. The definitions of two indices are as follows:

$$
\begin{gathered}
P S N R_{i}=10 * \log _{10} \frac{M N}{\sum_{x=1}^{M} \sum_{y=1}^{N}\left[\hat{u}_{i}(x, y)-u_{i}(x, y)\right]^{2}} \\
\text { Mean PSNR }=\frac{1}{B} \sum_{i=1}^{B} P S N R_{i} \\
\text { SSIM } M_{i}=\frac{\left(2 u_{u_{i}} u_{\hat{u}_{i}}+C_{1}\right)\left(2 \sigma_{u_{i}} u_{\hat{u}_{i}}+C_{2}\right)}{\left(u_{u_{i}}{ }^{2}+u_{\hat{u}_{i}}{ }^{2}+C_{1}\right)\left(\sigma_{u_{i}}{ }^{2}+\sigma_{\hat{u}_{i}}{ }^{2}+C_{2}\right)} \\
\text { Mean SSIM }=\frac{1}{B} \sum_{i=1}^{B} S S I M_{i}
\end{gathered}
$$


where $u_{i}$ and $\hat{u}_{i}$ represent the $i$ th band of the reference image and restored image, respectively. $u_{u_{i}}$ and $u_{\hat{u}_{i}}$ are the average values of image $u_{i}$ and $\hat{u}_{i}$, while $\sigma_{u_{i}}$ and $\sigma_{\hat{u}_{i}}$ are variances. And $M$ and $N$ are the height and width in the spatial region, respectively. Moreover, $B$ is the number of bands in spectrum region.

\subsection{Experiment results on AVIRIS Indian Pines}

In this section, the AVIRIS Indian Pines [32] are used in our experiment. The hyperspectral image was collected by the AVIRIS sensor over the Indian Pines region, Northwest Indiana, USA, in 1992. The scene was acquired over a mixed agricultural/forest area, with a size of $145 \times 145 \times 224$. The bands in the wavelength range from 0.2 to $2.5 \mathrm{um}$, nominal spectral resolution of $10 \mathrm{~nm}$. Furthermore, this image has a spatial resolution of $20 \mathrm{~m}$ per pixel and 16-bit radiometric resolution. It includes 16 ground-truth classes, most of which are different types of crops (e.g., corns, soybeans, and so on). For the preconditioning of the data, the gray values of each band of the HSI are normalized between $[0,1]$. In the experiment, we randomly adds $10 \%, 20 \%, 30 \%, 40 \%, 45 \%$ salt and pepper noise to all bands. At first, in order to find the best rank, we measure the relationship between the average peak signal-to-noise ratio and the target rank, displayed in Figure 2. From the figure, we can easily obtain that $\mathrm{N}=2$ is the best target rank in this data set. Similarly, we do the same operation on the ROSIS Pavia University Scene and Botswanna data set and besides, we obtain $\mathrm{N}=3$ is the best target rank in the two scenes, respectively.

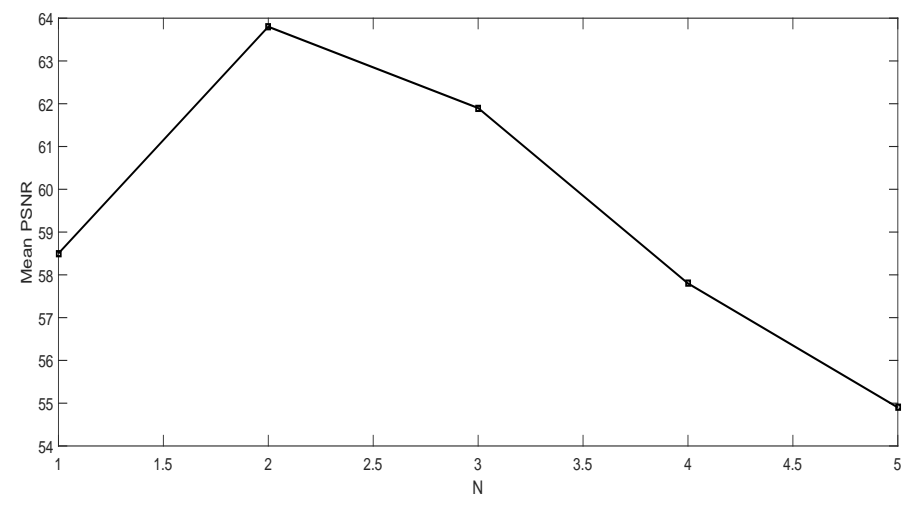

Figure 2: The relationship between Mean PSNR and the target rank $\mathrm{N}$ on Indian Pines 
In order to see an intuitive superpixel concept, we show the segmentation results on Indian Pines, shown in Figure 3. From this figure, it is not hard to see that the same type of sample is assigned to the same sub-block and samples around the border can be effectively separated. In this way, this will help us to get a better denoising result, and latter experiments also verify this view.

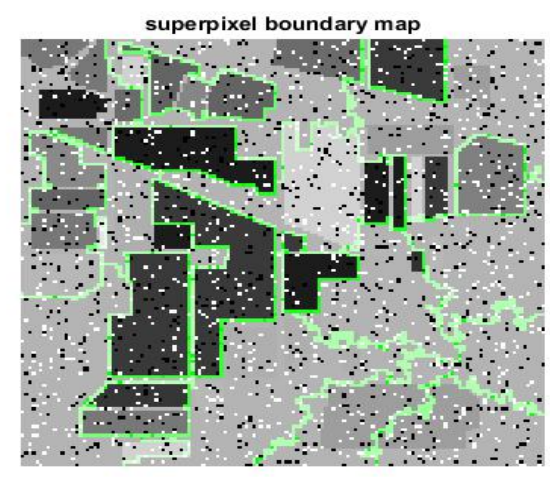

Figure 3: superpixel boundary map on Indian Pines.

Table 1 displays the results of mean PSNR with different methods under different ratio of noise. It can be seen clearly that, the mean PSNR of SS-PSSV is higher than others under $10 \%, 20 \%, 40 \%, 45 \%$ noise and PSSV achieves the best result under $30 \%$ noise, which indicates that PSSV and SS-PSSV outperform the other methods and are more effective in the respect of the denoising. Meanwhile, in Table 2, the results of mean SSIM of different methods with different ratio of noise are displayed. From this table, we can see that SS-PSSV can obtain better performance under $20 \%, 30 \%, 40 \%, 45 \%$ noise and PSSV achieves the best result under $10 \%$ noise, which suggests that SS-PSSV and PSSV are perfect and more effective in denoising. In addition, from Table 2, we easily get the conclusion that SS-PSSV has a more obvious advantage over others when noise ratio is bigger, that is to say, with the increase of noise, SS-PSSV's denoising effect is more obvious. Under $10 \%$ noise, PSSV obtains the best performance, which indicates PSSV model is very effective in hyperspectral field, and when superpixel is added into PSSV, SS-PSSV can get a bigger improvement, with increasing noise. So superpixel segmentation is very necessary for image process- 
ing. As we know, LRMR, RPCA, RPCAG and SS-LRR consider the clean sample as a low rank ingredient, and adopt the nuclear function to portray it. Notice that nuclear function minimizes the rank, but it does not fully utilize a prior target rank information about samples.

Table 1: Mean PSNR values of the restoration results with different restoration methods on Indian Pines

\begin{tabular}{cccccc}
\hline \hline Mean PSNR & $10 \%$ & $20 \%$ & $30 \%$ & $40 \%$ & $45 \%$ \\
\hline LRMR & 41.1566 & 37.2959 & 34.6609 & 32.3473 & 31.2417 \\
RPCA & 42.4305 & 37.4339 & 31.5867 & 26.5500 & 24.4990 \\
RPCAG & 54.6674 & 47.7350 & 41.2280 & 34.5390 & 31.0893 \\
SS-LRR & 45.5827 & 42.2679 & 38.1975 & 36.1948 & 34.0037 \\
PSSV & 47.0909 & 45.1279 & $\mathbf{4 4 . 1 6 2 6}$ & 41.2269 & 37.4463 \\
SS-PSSV & $\mathbf{5 5 . 5 3 2 4}$ & $\mathbf{5 0 . 3 4 6 9}$ & 43.2180 & $\mathbf{4 1 . 3 6 1 1}$ & $\mathbf{3 7 . 9 9 4 7}$ \\
\hline \hline
\end{tabular}

Table 2: Mean SSIM values of the restoration results with different restoration methods on Indian Pines

\begin{tabular}{cccccc}
\hline \hline Mean SSIM & $10 \%$ & $20 \%$ & $30 \%$ & $40 \%$ & $45 \%$ \\
\hline LRMR & 0.9770 & 0.9498 & 0.9172 & 0.8766 & 0.8494 \\
RPCA & 0.9946 & 0.9849 & 0.9362 & 0.8223 & 0.7537 \\
RPCAG & 0.9955 & 0.9934 & 0.9918 & 0.9719 & 0.9479 \\
SS-LRR & 0.9949 & 0.9933 & 0.9885 & 0.9727 & 0.9359 \\
PSSV & $\mathbf{0 . 9 9 6 4}$ & 0.9935 & 0.9919 & 0.9834 & 0.9601 \\
SS-PSSV & 0.9955 & $\mathbf{0 . 9 9 3 7}$ & $\mathbf{0 . 9 9 5 2}$ & $\mathbf{0 . 9 8 9 8}$ & $\mathbf{0 . 9 8 1 5}$ \\
\hline \hline
\end{tabular}



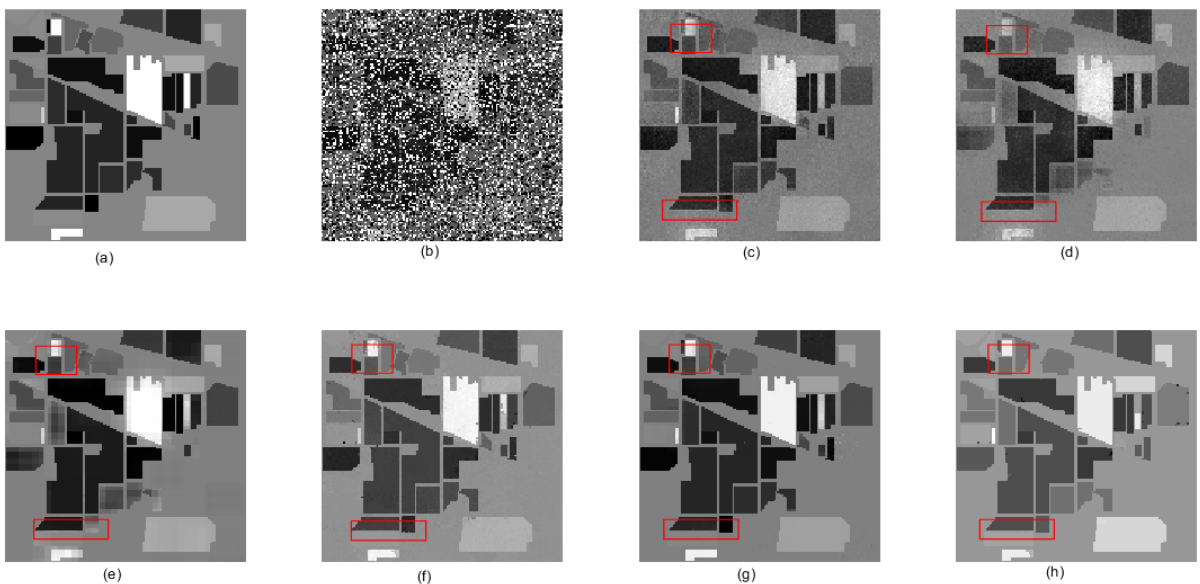

Figure 4: Restoration results using different methods on Indian Pines: (a) Original band 1, (b) noisy band with ratio of $40 \%$ salt and pepper noise, (c) LRMR, (d) RPCA, (e) RPCAG, (f) SS-LRR, (g) PSSV, (h) SS-PSSV.

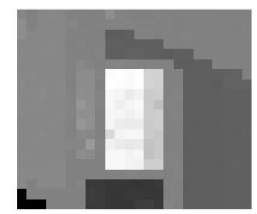

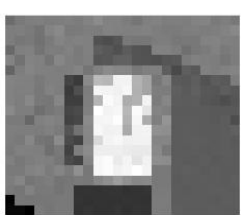

(f)

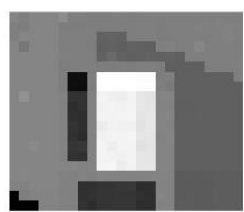

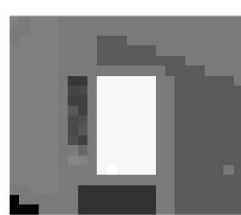

Figure 5: A partial enlargement of the red box in Figure 4 with (e) RPCAG, (f) SS-LRR, (g) PSSV, (h) SS-PSSV. 

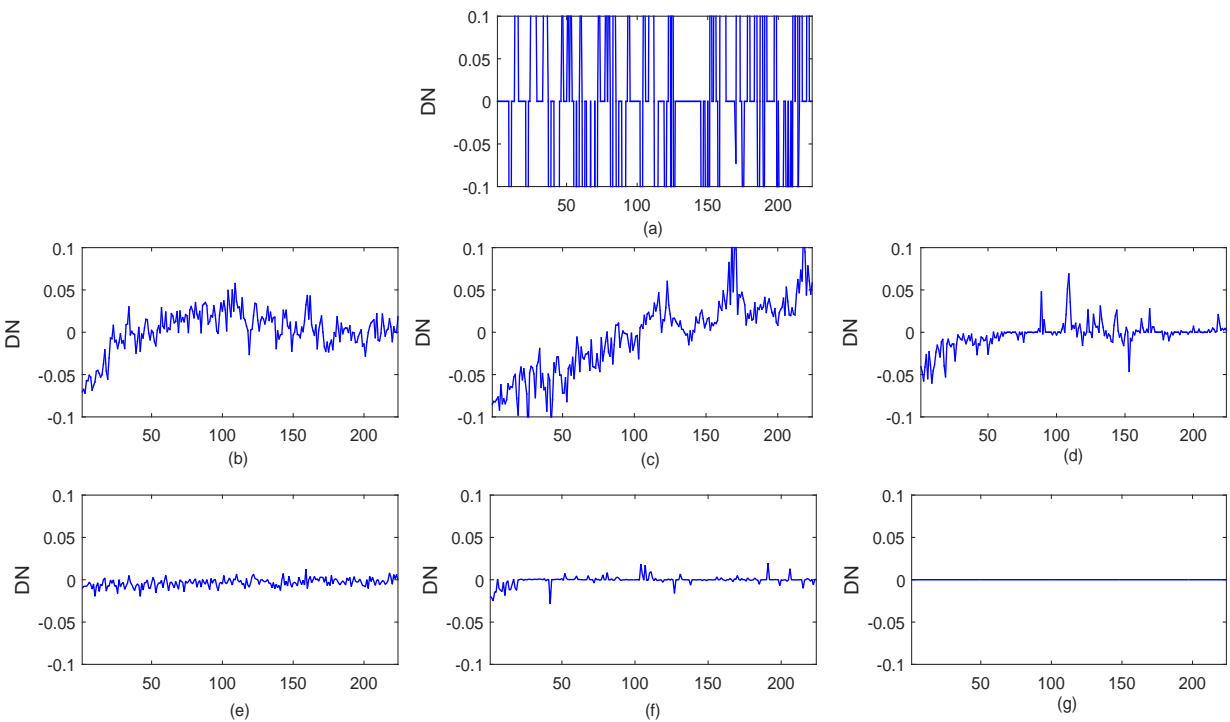

Figure 6: Difference between the noise-free spectrum and the restoration results of pixel $(60,75)$ with ratio of $40 \%$ salt and pepper noise on Indian Pines: (a) noisy, (b) LRMR, (c) RPCA, (d) RPCAG, (e) SS-LRR, (f) PSSV, (g) SS-PSSV.

In the following, we will show some denoised images, and Figure 4 displays the restoration performance under $40 \%$ salt and pepper noise for first band. The picture (a) is clean image, and picture (b) is with $40 \%$ salt and pepper noise. The pictures (c) to (h) exhibit the restoration results with different methods, respectively. From the Figure 4, we can find LRMR, RPCA do not remove the noise wholly, and although RPCAG remove the most noise, there is a picture overlapping problem as you see while the restoration from SS-LRR have a fact of information loss, which can be observed easily especially in red rectangle box. But with PSSV and SS-PSSV, the noise is basically removed effectively while retaining most of the picture information at the same time. In order to be able to see a clearer denoising effect, we make a partial enlargement of the denoised image in Figure 5 with methods including RPCAG, SS-LRR, PSSV, SS-PSSV. From this figure, we see clearly that (e) and (f) still exist much noise. And although compared with the previous two images, (g) removes most of the noise, there 
is part of the residual. From (h), we can easily get that SS-PSSV achieves the best denoising performance, which shows that the combination of superpixel segmentation and PSSV makes sense.

Furthermore, the difference between the noise-free spectrum and the restoration results in the spectral signatures at $(60,75)$ under $40 \%$ salt and pepper noise are shown in Figure 6. From the Figure 6, we can easily see the difference is bigger in all bands from (b),(c),(d),(e), while SS-PSSV is consistent with the original information, which suggests that SS-PSSV has an advantage over others. Because SS-PSSV first adopt the superpixel segmentation to make the same class pixel into an superpixel, and so it can be handled as a whole.

Furthermore, in order to avoid accidental effects, we exhibit the horizonal and vertical profiles of band 50 at pixel $(20,30)$, respectively, where horizonal profiles is a vector on band 50 with the second coordinate being 30 in spatial domain, and vertical profiles is also a vector on band 50 with the first coordinate being 20 in spatial domain. In (c) and (d) of Figure 7, we easily see that LRMR and RPCA perform poorly. And although compared with the two model, performances of RPCAG and SS-LRR are better, and still we can see there exist thrill in column numbers from 30 to 140 especially in (f), and this phenomenon can also be found in Figure 8. In Figure 8, performances of LRMR, RPCA, SS-LRR are bad in most row number, and compared with the models mentioned, RPCAG performs better. However, there is a slight jitter in (e) in row numbers from 20 to 80 . Of course, PSSV also has some glitches while SS-PSSV perform well in almost all bands.

Finally, in order to get more perspective and more comprehensive comparison among several methods, PSNR values with different approaches on each band are displayed in Figure 9, which is under 45\% salt and pepper noise. From this figure, we can see that SS-PSSV and PSSV are more high than others in most bands although there is several rapid decline. Because we firstly adopt the superpixel segmentation, and then make full use of target rank to remove noise for each superpixel. Furthermore, SSIM values on every band are displayed in Figure 10. The larger SSIM value is, the better denoising quality is. From the figure, we can easily see that PSSV and SS-PSSV achieve better outcome. 

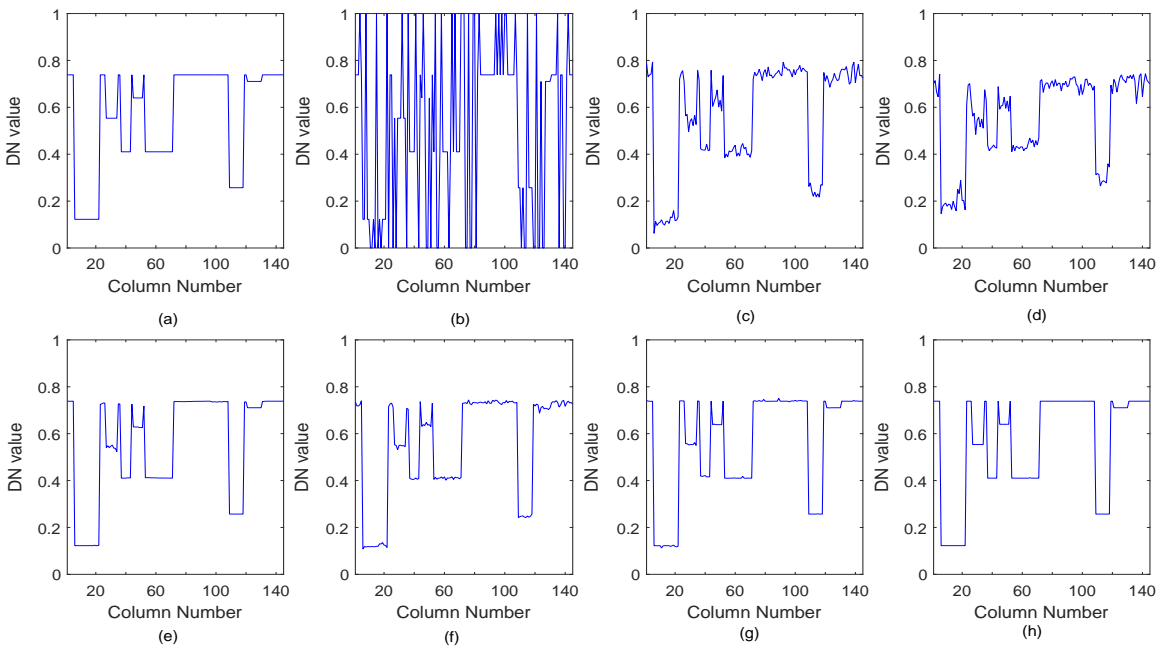

Figure 7: Horizontal profiles of band 50 at pixel $(20,30)$ before and after restoration on Indian Pines: (a) Original, (b) noisy, (c) LRMR, (d) RPCA, (e) RPCAG, (f) SS-LRR, (g) PSSV, (h) SS-PSSV.

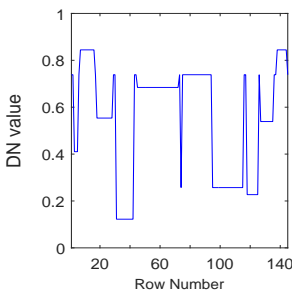

(a)
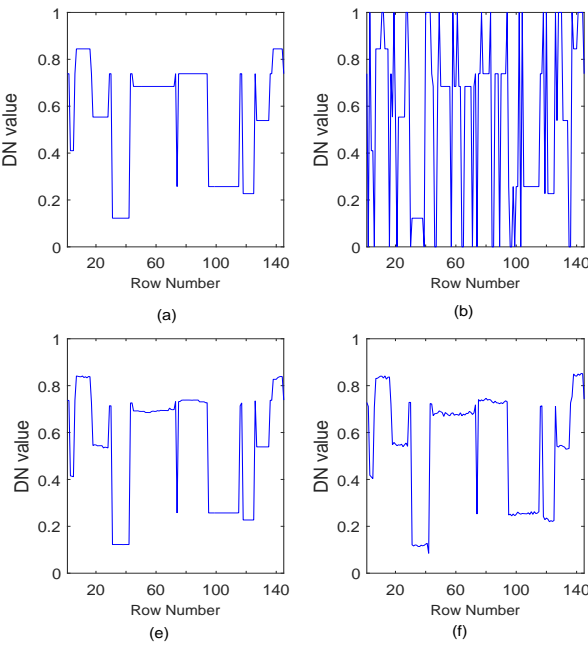

(b)

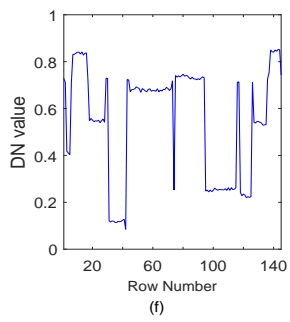

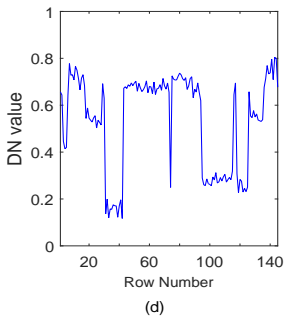
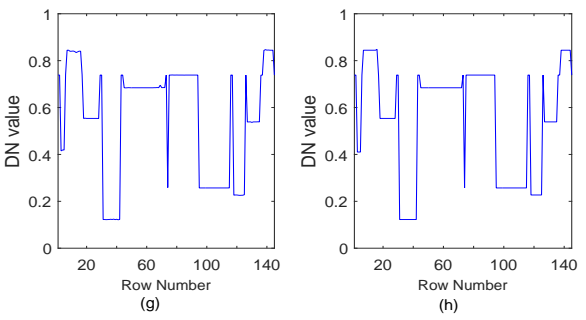

Figure 8: Vertical profiles of band 50 at pixel $(20,30)$ before and after restoration on Indian Pines: (a) Original, (b) noisy, (c) LRMR, (d) RPCA, (e) RPCAG, (f) SS-LRR, (g) PSSV, (h) SS-PSSV. 


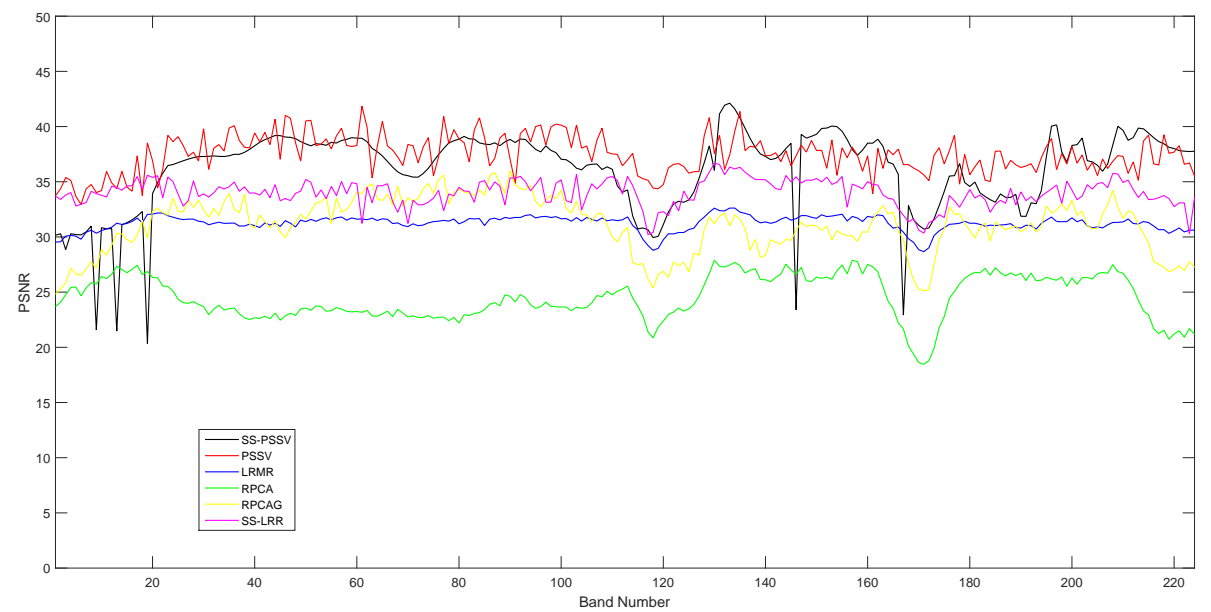

Figure 9: PSNR values of each band of the $45 \%$ noise experimental results with the different restoration methods on Indian Pines

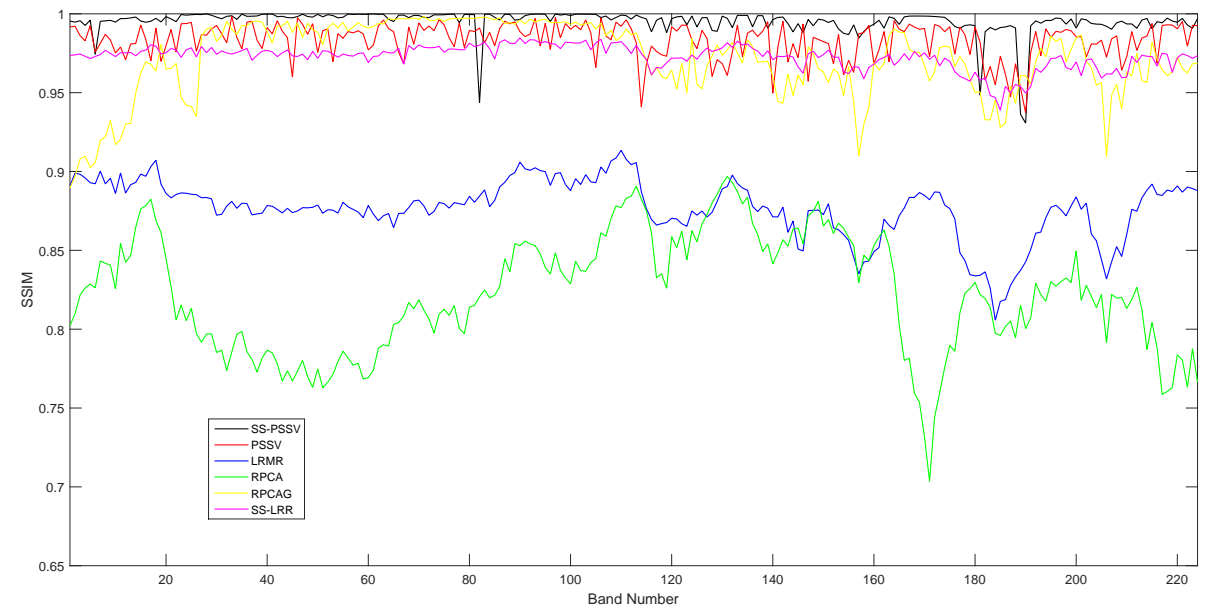

Figure 10: SSIM values of each band of the $40 \%$ noise experimental results with the different restoration methods on Indian Pines

In summary, AVIRIS Indian Pines contains a variety of crops and the edge between crops is a curve, not a regular straight line. So if we utilize conventional rectangular block, not using superpixel segmentation, we will not be able to deal well with samples that are around the edge. For example, PSSV segment images with conventional rectangular block, and from the simulation results, we find that SS-PSSV has a better 
advantage over PSSV in most experimental results. So in this paper, we use superpixel segmentation skillfully, in this way, we can make same class of pixels into superpixel as much as possibly. Finally, we adopt PSSV to every superpixel block which can make full use of prior knowledge about target rank.

\subsection{Experiment results on the ROSIS Pavia University Scene}

In this subject, the ROSIS Pavia University scene is adopted to test the performance of the proposed method. The data were collected by using the Reflective Optics System Imaging Spectrometer sensor on the urban area of the University of Pavia, Italy [33]. This image has a size of $610 * 340$ in pixels with a spatial resolution of $1.3 \mathrm{~m}$ per pixel. It altogether includes 115 spectral bands ranging from 0.43 to $0.86 u m$ in spectrum. After discarding 12 noisy and water absorption bands, 103 bands are retained in our experiment. The ground truth is classified into 9 mutually exclusive classes including Trees, Metal sheets and so on. For the preconditioning of the data, the gray values of each band of the HSI is normalized between $[0,1]$. In experiments, we randomly adds $10 \%, 20 \%, 30 \%, 40 \%, 45 \%$ salt and pepper noise to all bands.

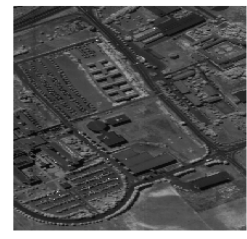

(a)

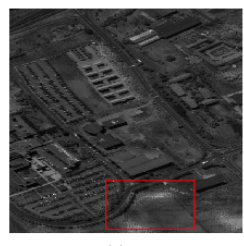

(e)

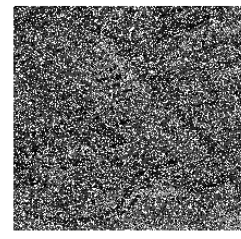

(b)

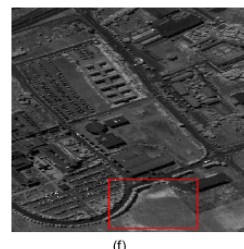

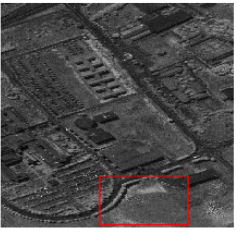

(c)

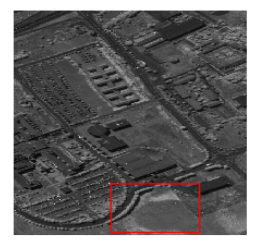

(g)

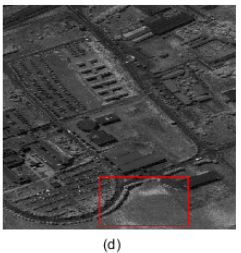

(d)

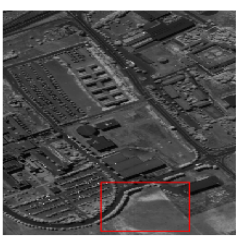

(h)

Figure 11: Restoration results using different methods on Pavia University: (a) Original band 101, (b) noisy band with ratio of $40 \%$ salt and pepper noise, (c) LRMR, (d) RPCA, (e) RPCAG, (f) SS-LRR, (g) PSSV, (h) SS-PSSV. 

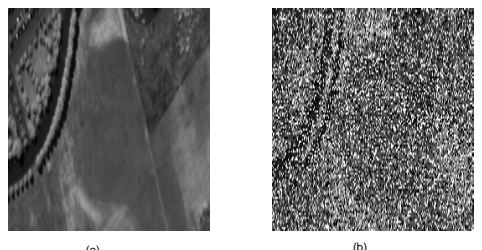

(b)

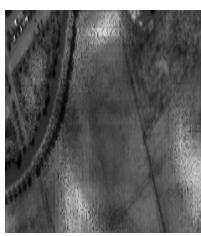

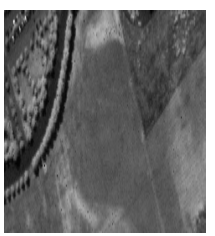
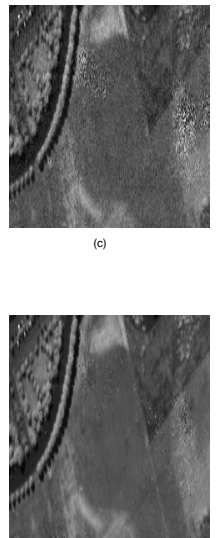
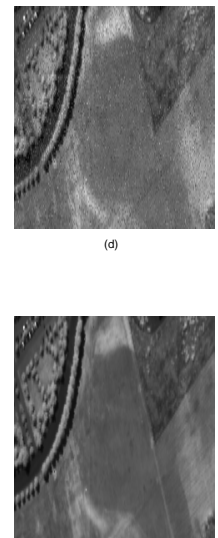

Figure 12: A partial enlargement of the red box in Figure 11.

Table 3: mean PSNR values of the restoration results with different restoration methods on Pavia University

\begin{tabular}{cccccc}
\hline \hline mean PSNR & $10 \%$ & $20 \%$ & $30 \%$ & $40 \%$ & $45 \%$ \\
\hline LRMR & 36.7825 & 33.6803 & 31.3048 & 29.2001 & 28.1391 \\
RPCA & 38.3142 & 35.4068 & 32.3950 & 28.8795 & 26.9263 \\
RPCAG & 38.4600 & 37.5966 & 35.7621 & 31.0703 & 27.8292 \\
SS-LRR & 36.3178 & 35.2218 & 34.0092 & 32.5643 & 28.2567 \\
PSSV & 41.8244 & 38.4779 & 36.4456 & 33.7284 & 30.6439 \\
SS-PSSV & $\mathbf{4 2 . 1 3 5 7}$ & $\mathbf{4 0 . 1 4 8 1}$ & $\mathbf{3 8 . 1 6 0 6}$ & $\mathbf{3 7 . 2 1 5 7}$ & $\mathbf{3 4 . 3 3 8 8}$ \\
\hline \hline
\end{tabular}

To measure the performance, mean PSNR and mean SSIM among 103 bands are calculated. So Table 3 and Table 4 display the results of mean PSNR and mean SSIM in ROSIS Pavia University Scene. From the table, it can be seen clearly that, SSPSSV achieves the best performance and PSSV get second best result. So superpixel segmentation and PSSV undoubtedly open up a new direction for noise removal. 
Table 4: mean SSIM values of the restoration results with different restoration methods on Pavia University

\begin{tabular}{cccccc}
\hline \hline mean SSIM & $10 \%$ & $20 \%$ & $30 \%$ & $40 \%$ & $45 \%$ \\
\hline LRMR & 0.9366 & 0.8875 & 0.8323 & 0.7651 & 0.7239 \\
RPCA & 0.9725 & 0.9533 & 0.9055 & 0.8004 & 0.7183 \\
RPCAG & 0.9762 & 0.9617 & 0.9527 & 0.8621 & 0.8028 \\
SS-LRR & 0.9628 & 0.9537 & 0.9427 & 0.9228 & 0.8213 \\
PSSV & 0.9764 & 0.9617 & 0.9483 & 0.9111 & 0.8361 \\
SS-PSSV & $\mathbf{0 . 9 7 8 1}$ & $\mathbf{0 . 9 7 3 8}$ & $\mathbf{0 . 9 6 6 8}$ & $\mathbf{0 . 9 6 2 8}$ & $\mathbf{0 . 9 5 0 2}$ \\
\hline \hline
\end{tabular}

In order to make a intuitive comparison, we displays restoration image on band 101 under $40 \%$ salt and pepper noise in Figure 11. ROSIS Pavia University Scene contains tree and so on, so if effective methods are not utilized to segment image, it will be hard to get a good denoising result. It is obvious that traditional segmentation methods can not effectively divide the complex image, so in this paper, we decide to adopt superpixel segmentation, because it can handle border information well. Picture (a) is an original image, and (b) shows noisy image with $40 \%$ salt and pepper noise. The pictures of (c) to (h) display restoration results with different models, respectively. From Figure 11, we can see that in the restorations with LRMR and RPCA, there has still noise not been removed. Similarly, denoised image of RPCAG also performs poorly and recovered image is blurred, so edge information is not preserved completely which can be observed easily in red rectangle box. Simultaneously, the image of the denoising with SS-LRR also has the phenomenon that edge information is incomplete. Only in PSSV and SS-PSSV, the recovered image has ability to preserve edge of image and effective removal of noise. In order to see more clearly, we have partially enlarged the images, as shown in Figure 12.

In order to further explore the characteristics of hyperspectral image, we draw the difference between the noise-free spectrum and the restoration at $(300,120)$ under $40 \%$ salt and pepper noise. From Figure 13, we can see the difference from SS-PSSV is smallest. 

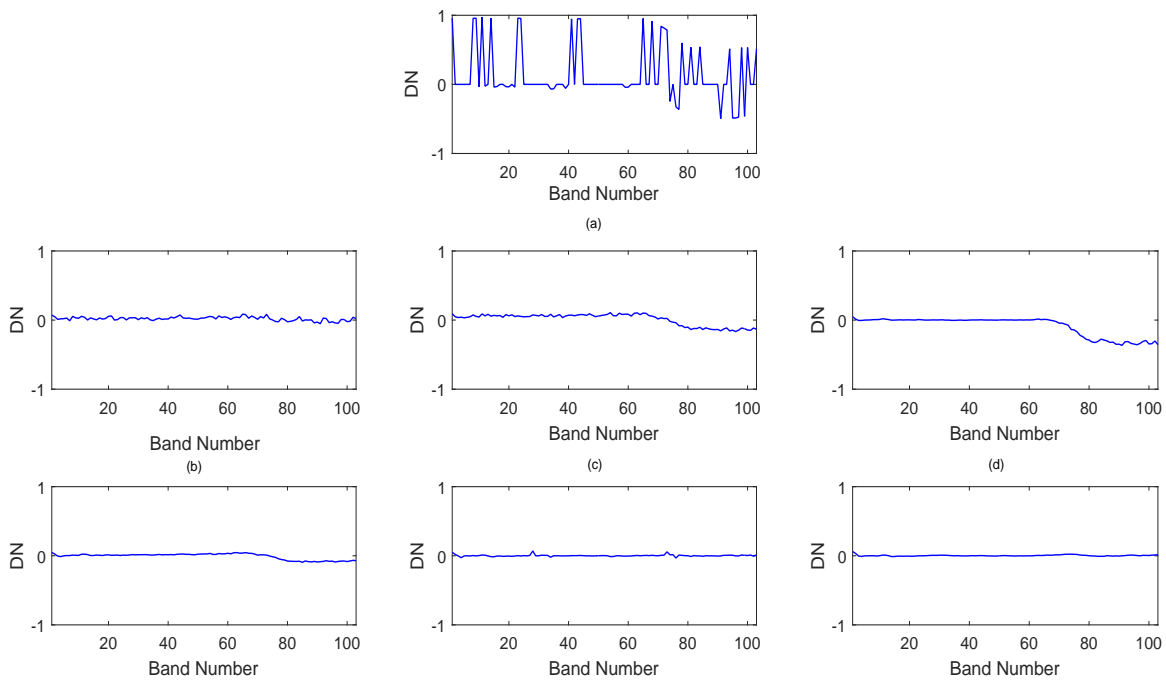

Figure 13: Difference between the noise-free spectrum and the restoration results of pixel $(300,120)$ with ratio of $40 \%$ salt and pepper noise on Pavia University: (a) noisy, (b) LRMR, (c) RPCA, (d) RPCAG, (e) SS-LRR, (f) PSSV, (g) SS-PSSV.

To further test PSSV and SS-PSSV, we calculate horizonal and vertical profiles of band 96 at pixel $(100,100)$, respectively. From Figure 14, we easily see that there is a serious glitch on the curve in contrast models, especially more obvious in pictures (c), (d) and (e), and this fact suggests noise is not completely removed. However, there is no such problem in the curve of picture (g) and (h). In addition, the longitudinal curve in Figure 15 also verifies that our argument is correct. In Figure 15, there is a more serious glitch on the curve on contrast approaches and still there is no such problem in the curve of PSSV and SS-PSSV, that is to say, the results with PSSV and SS-PSSV are more similar to the original in most bands, which undoubtedly validates the fact that superpixel segmentation and PSSV provide a new perspective of image processing.

For a more comprehensive result, the PSNR and SSIM values on all bands of restoration with different approaches are displayed in Figure 16 and Figure 17, respectively. From Figure 16, we can clearly see that the curve of PSSV or SS-PSSV is 
always above the curves of the other methods in almost all bands and Figure 17 exists the same solution as Figure 16, which indicates the effectiveness of superpixel segmentation for removing the noise and PSSV is also beneficial to denoise in hyperspectral images.
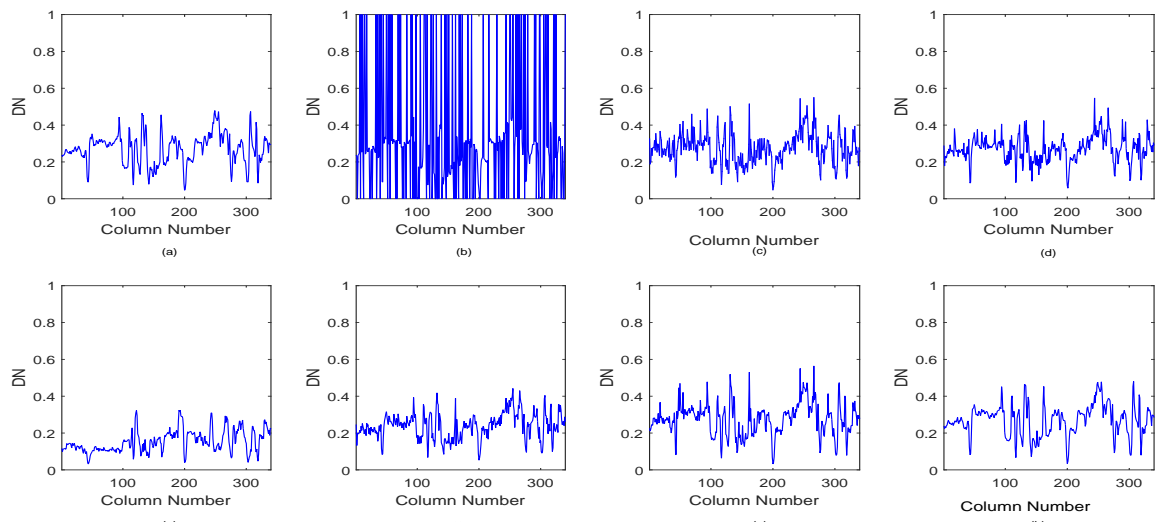

Figure 14: Horizontal profiles of band 96 at pixel $(100,100)$ before and after restoration on Pavia University: (a) Original, (b) noisy, (c) LRMR, (d) RPCA, (e) RPCAG, (f) SS-LRR, (g) PSSV, (h) SS-PSSV.

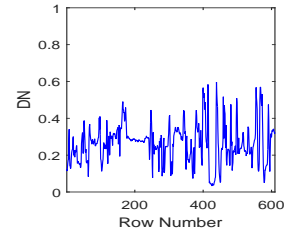

(a)

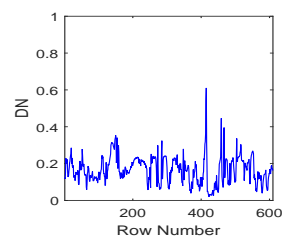

(e)
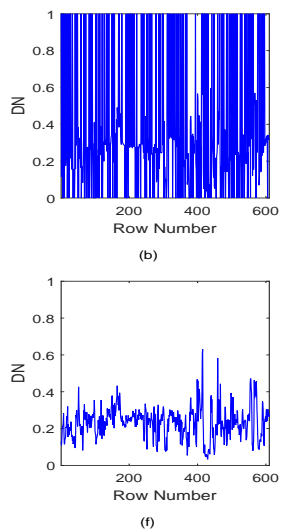
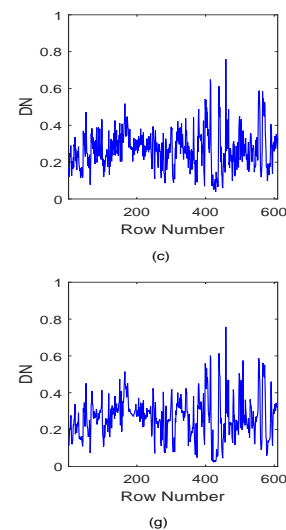
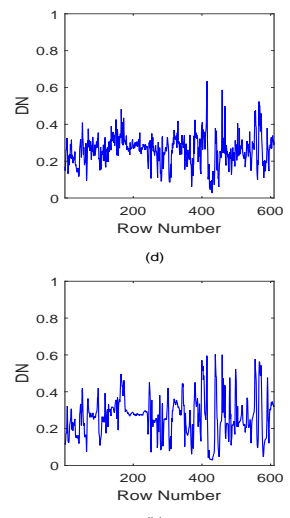

(h)

Figure 15: Vertical profiles of band 96 at pixel $(100,100)$ before and after restoration on Pavia University: (a) Original, (b) noisy, (c) LRMR, (d) RPCA, (e) RPCAG, (f) SS-LRR, (g) PSSV, (h) SS-PSSV. 
Table 5: mean PSNR values of the restoration results with different restoration methods on Pavia University

\begin{tabular}{cccccc}
\hline \hline mean PSNR & $10 \%$ & $20 \%$ & $30 \%$ & $40 \%$ & $45 \%$ \\
\hline LRMR & 36.2991 & 33.8515 & 31.9306 & 30.1682 & 29.2828 \\
RPCA & 37.3870 & 36.1057 & 33.7716 & 30.0776 & 27.9129 \\
RPCAG & 37.9592 & 37.5454 & 34.5579 & 30.9087 & 28.9145 \\
SS-LRR & 36.2243 & 35.5279 & 34.7330 & 33.6713 & 29.7033 \\
PSSV & $\mathbf{3 8 . 8 7 5 0}$ & 38.1106 & $\mathbf{3 7 . 0 4 0 2}$ & $\mathbf{3 4 . 7 4 4 2}$ & 32.0922 \\
SS-PSSV & 38.2876 & $\mathbf{3 8 . 2 3 7 2}$ & 35.7312 & 34.2281 & $\mathbf{3 3 . 7 1 4 7}$ \\
\hline \hline
\end{tabular}

Table 6: mean SSIM values of the restoration results with different restoration methods on Pavia University

\begin{tabular}{cccccc}
\hline \hline mean SSIM & $10 \%$ & $20 \%$ & $30 \%$ & $40 \%$ & $45 \%$ \\
\hline LRMR & 0.9465 & 0.9134 & 0.8786 & 0.8357 & 0.8096 \\
RPCA & 0.9677 & 0.9606 & 0.9385 & 0.8739 & 0.8231 \\
RPCAG & 0.9675 & 0.9638 & 0.9539 & 0.9273 & 0.8937 \\
SS-LRR & 0.9606 & 0.9554 & 0.9486 & 0.9347 & 0.8829 \\
PSSV & 0.9676 & 0.9644 & 0.9574 & 0.9348 & 0.8940 \\
SS-PSSV & $\mathbf{0 . 9 6 8 1}$ & $\mathbf{0 . 9 6 9 7}$ & $\mathbf{0 . 9 6 2 2}$ & $\mathbf{0 . 9 4 3 4}$ & $\mathbf{0 . 9 4 0 0}$ \\
\hline \hline
\end{tabular}

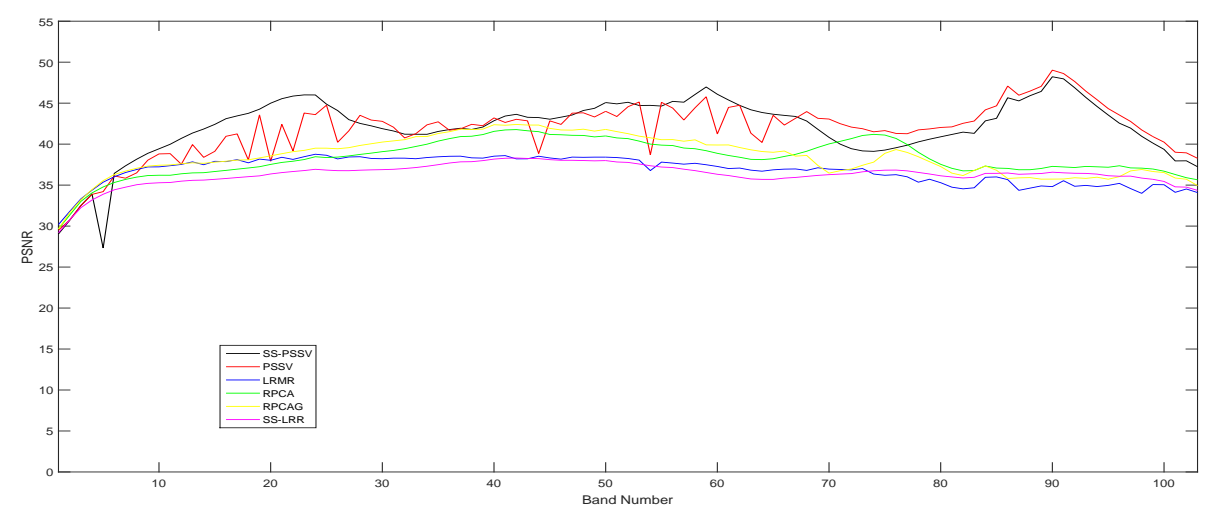

Figure 16: PSNR values of each band of the $10 \%$ noise experimental results with the different restoration methods on Pavia University 


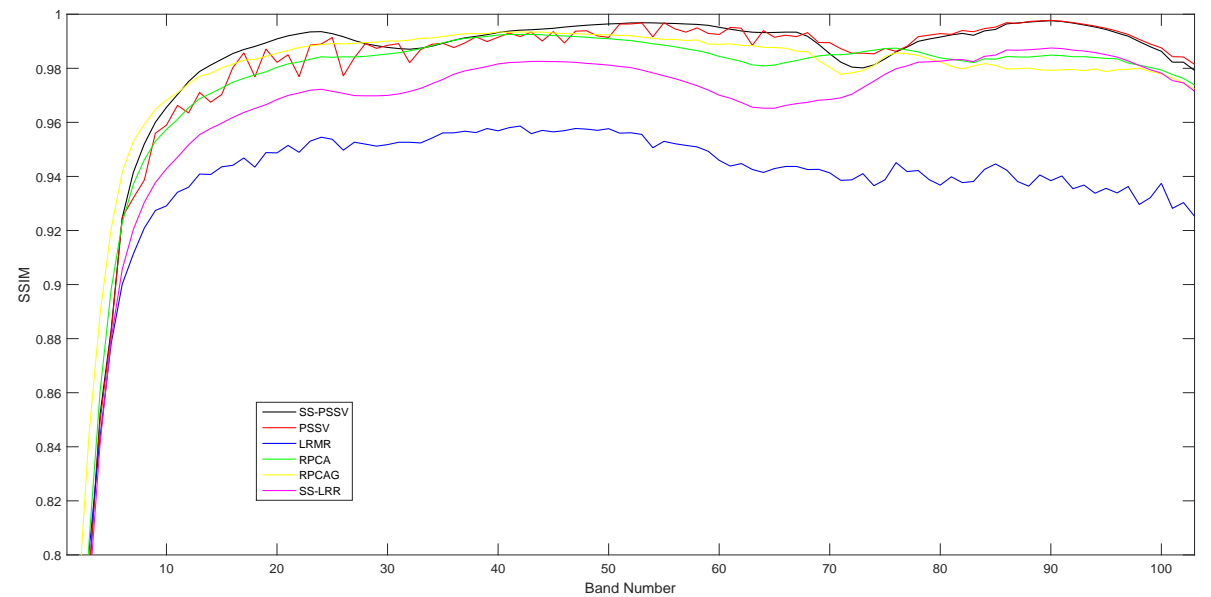

Figure 17: SSIM values of each band of the $10 \%$ noise experimental results with the different restoration methods on Pavia University

\subsection{Experiment results on the Botswanna data set}

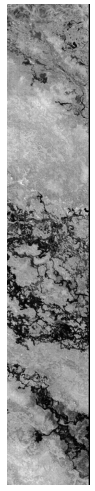

(a)

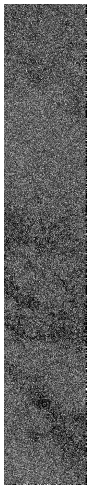

(b)

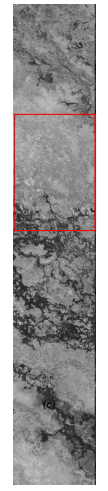

(c)

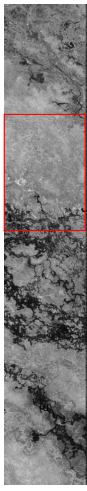

(d)

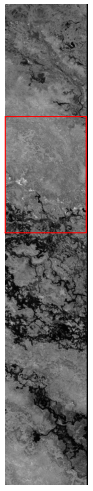

(e)

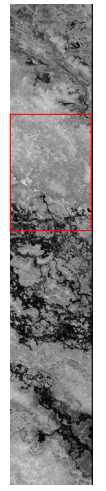

(f)

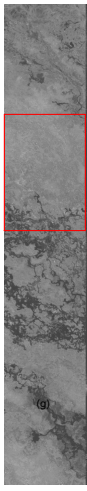

(g)

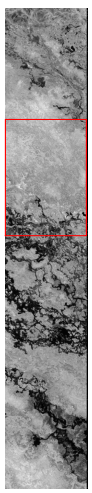

(h)

Figure 18: Restoration results using different methods on BOT: (a) Original band 100, (b) noisy band with ratio of $30 \%$ salt and pepper noise, (c) LRMR, (d) RPCA, (e) RPCAG, (f) SS-LRR, (g) PSSV, (h) SS-PSSV. 


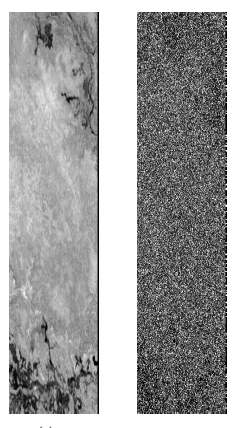

(b)

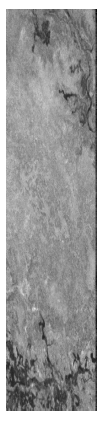

(c)

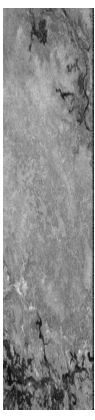

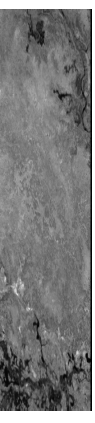

(e)

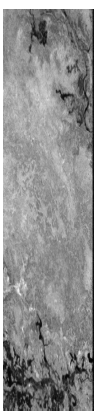

(1)

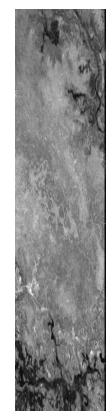

(g)

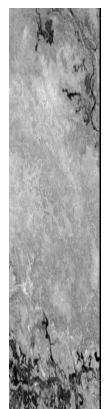

(n)

Figure 19: A partial enlargement of the red box in Figure 18.

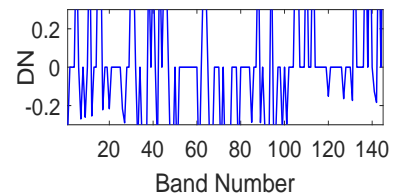

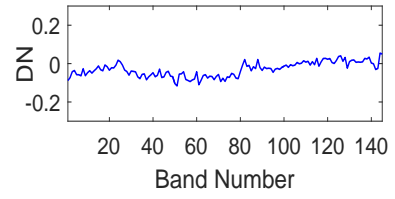

(b)

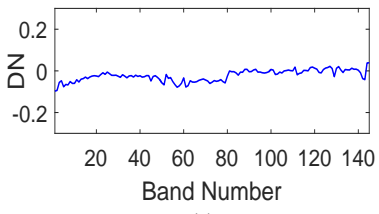

(e) (a)

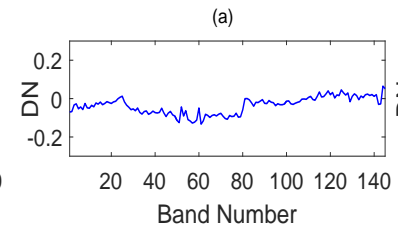

(c)

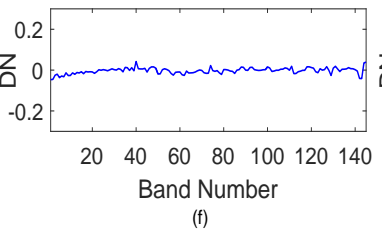

(f)

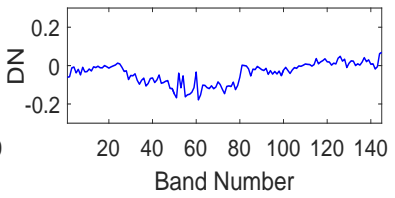

(d)

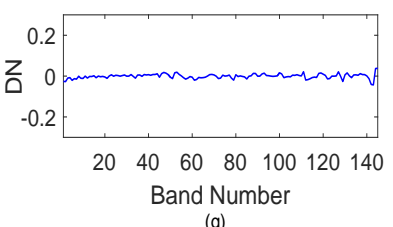

(g)

Figure 20: Difference between the noise-free spectrum and the restoration results of pixel $(60,75)$ with ratio of $40 \%$ salt and pepper noise on BOT: (a) noisy, (b) LRMR, (c) RPCA, (d) RPCAG, (e) SS-LRR, (f) PSSV, (g) SS-PSSV. 

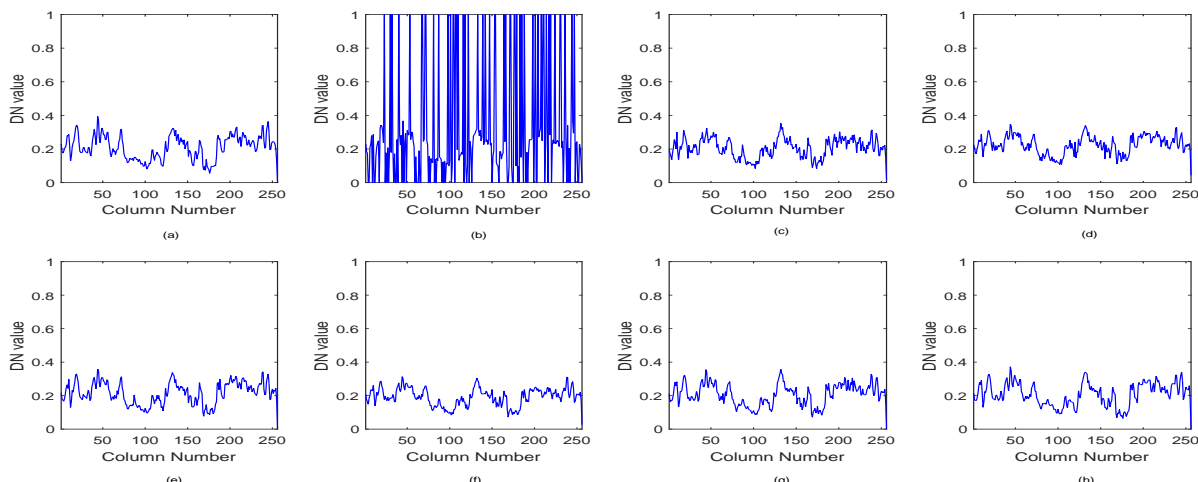

Figure 21: Horizontal profiles of band 122 at pixel $(100,80)$ before and after restoration on BOT: (a) Original, (b) noisy, (c) LRMR, (d) RPCA, (e) RPCAG, (f) SS-LRR, (g) PSSV, (h) SS-PSSV.
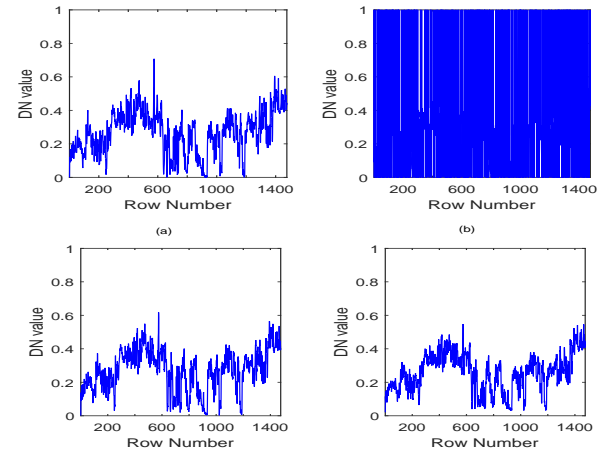

(e)

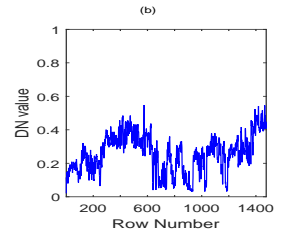

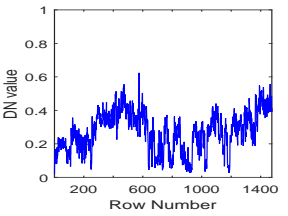
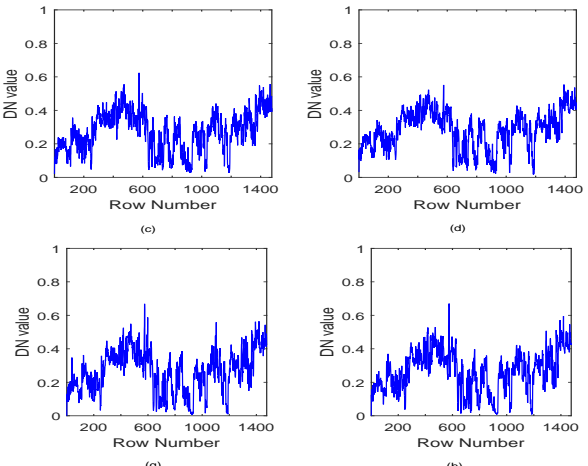

(h)

Figure 22: Vertical profiles of band 122 at pixel $(100,80)$ before and after restoration on BOT: (a) Original, (b) noisy, (c) LRMR, (d) RPCA, (e) RPCAG, (f) SS-LRR, (g) PSSV, (h) SS-PSSV.

In our experiment, the Botswanna data set [34] is adopted, and the hyperspectral image was acquired across Okavango Delta, Botswana(BOT). The hyperspectral data includes two major ecosystem components defined by absence or presence of flooding, namely: upland and wetland. The data set was collected in 2001 and has a size of $1476 * 256$ with a spatial resolution of $30 \mathrm{~m}$ in pixels, and there are altogether 1580 labeled data points and includes 242 spectral bands ranging from 357 to $2576 \mathrm{~nm}$, with 
a hyperspectral resolution of $10 \mathrm{~nm}$. After discarding some noisy and water absorption bands, 145 bands are retained for our experiment, and the ground truth is classified into 9 mutually exclusive classes including Water, Primary Floodplain, Riparian and so on.

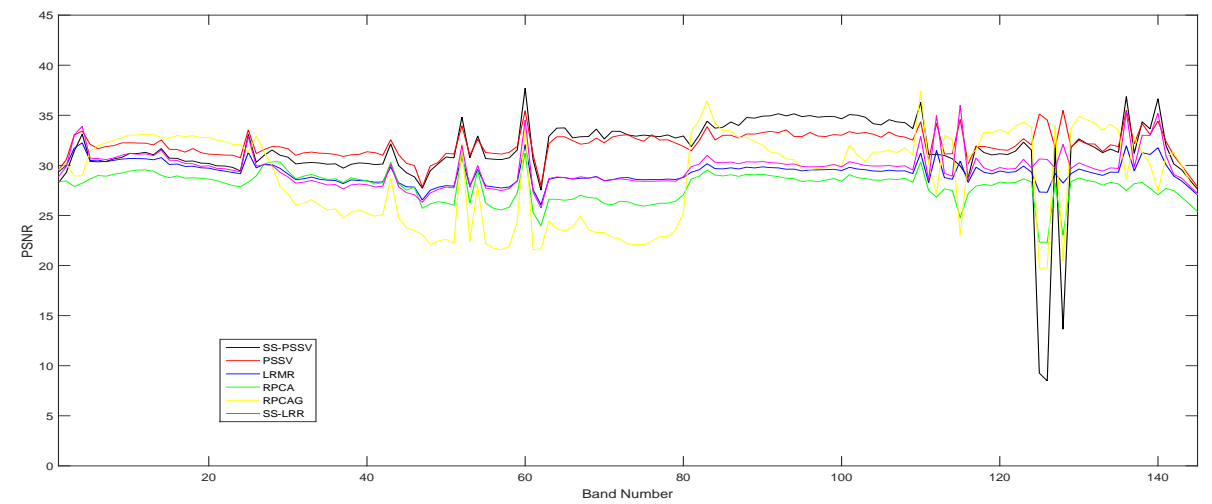

Figure 23: PSNR values of each band of the $45 \%$ noise experimental results with the different restoration methods on BOT.

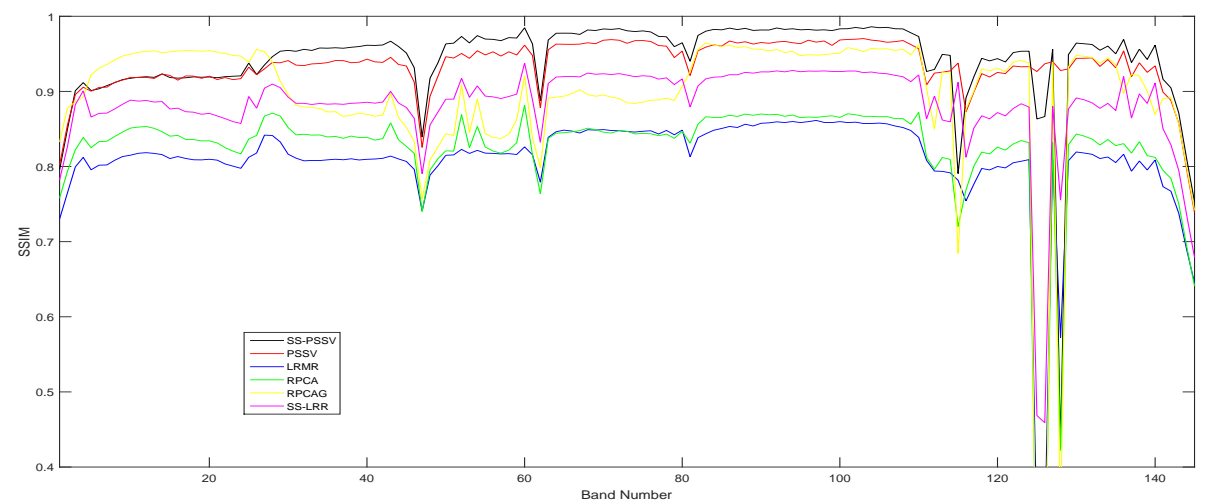

Figure 24: SSIM values of each band of the $45 \%$ noise experimental results with the different restoration methods on BOT.

We first calculate mean PSNR and mean SSIM, which are displayed on Table 5 and Table 6 respectively, and two tables show the performance with several methods under different ratio of noise. From Table 5, we can see that under 20\%, $45 \%$ noise, SSPSSV achieves the best performance, and under 10\%,30\%, 40\% noise, PSSV achieves the best performance. And to give visual comparisons, the recovery images are shown. 
In Figure 18, we display recovery images on band 100 with $30 \%$ salt and pepper noise with different models. The picture (a) displays original image on band 100, and picture (b) shows noisy graphic with ratio of $30 \%$ salt and pepper noise. Pictures (c) to (h) exhibit the restorations with different methods, respectively. From these comparisons, we can see that SS-PSSV can recover a more vivid image, which can be observed easily in red rectangle box. And an enlarged image of the red box will be shown in Figure 19.

In order to have a deeper and comprehensive understanding on spectral signatures, we observe the difference between the noise-free spectrum and the restoration at $(60,75)$ under $40 \%$ salt and pepper noise. From Figure 20, we can clearly see that SS-PSSV has an big advantage over others. In order to have a more in-depth exploration of botswanna data set, we records the horizontal profiles and the vertical profiles of band 122 at pixel $(100,80)$, and the curves are displayed in Figure 21 and Figure 22, respectively. From Figure 21, we can see that contrast methods do not handle with noise well at column numbers between 240 and 250, while PSSV and SS-PSSV do it. The same situation appear in Figure 22, because LRMR, RPCA, RPCAG, SS-LRR can not remove noise well around row number 600, while PSSV and SS-PSSV can do.

Finally, in order to make a full understanding on all bands, PSNR and SSIM values of restorations with different approaches on each band are displayed in Figure 23 and Figure 24, respectively. It can be clearly seen that PSNR and SSIM values with PSSV and SS-PSSV are higher than others in most bands, which indicates the effectiveness of superpixel segmentation and PSSV for removing noise. In summary, superpixel segmentation allows the same type of samples are divided into the same sub-block as possible, then PSSV takes full advantage of the priori knowledge of the target rank. When we combine the two effectively, one should get better denoising effect in theory.

\section{Conclusions}

In this work, we combine superpixel segmentation and PSSV, named as SS-PSSV. The superpixel segmentation can obtain homogeneous regions, which can make full use of both hyperspectral and spatial information of HSIs and PSSV makes full use of the prior knowledge on each homogenous region, which can help remove noise effectively. 
Experiments on three HSI data sets have been conducted to demonstrate that PSSV and SS-PSSV outperforms other comparing methods in the field of hyperspectral denoising.

\section{References}

[1] H. Saari, A. Akuj?rvi, C. Holmlund, H. Ojanen, J. Kaivosoja, A. Nissinen, O. Niemel?inen, Visible, very near ir and short wave ir hyperspectral drone imaging system for agriculture and natural water applications, ISPRS - International Archives of the Photogrammetry, Remote Sensing and Spatial Information Sciences XLII-3/W3 (2017) 165-170.

[2] C. Winkens, V. Kobelt, D. Paulus, Robust features for snapshot hyperspectral terrain-classification, in: International Conference on Computer Analysis of Images and Patterns, 2017, pp. 16-27.

[3] Y. Sun, Y. Zhao, K. Qin, F. Tian, P. Liu, New identification of sericite subclass minerals using airborne hyperspectral data in the xitan region of gansu province and its significance in gold ore prospecting, Acta Geologica Sinica 92 (1) (2018) $426-427$.

[4] N. Ma, Y. Peng, S. Wang, P. Leong, An unsupervised deep hyperspectral anomaly detector., Sensors 18 (3) (2018) 693.

[5] A. V. Vo, L. Truong-Hong, D. F. Laefer, D. Tiede, S. DOleire-Oltmanns, A. Baraldi, M. Shimoni, G. Moser, D. Tuia, Processing of extremely high resolution lidar and rgb data: Outcome of the 2015 ieee grss data fusion contestłpart b: 3-d contest, IEEE Journal of Selected Topics in Applied Earth Observations and Remote Sensing 9 (12) (2017) 5560-5575.

[6] G. Chen, S.-E. Qian, Denoising of hyperspectral imagery using principal component analysis and wavelet shrinkage, IEEE Transactions on Geoscience and remote sensing 49 (3) (2011) 973-980. 
[7] Q. Yuan, L. Zhang, H. Shen, Hyperspectral image denoising employing a spectral-spatial adaptive total variation model, IEEE Transactions on Geoscience and Remote Sensing 50 (10) (2012) 3660-3677.

[8] H. Othman, S.-E. Qian, Noise reduction of hyperspectral imagery using hybrid spatial-spectral derivative-domain wavelet shrinkage, IEEE Transactions on Geoscience and Remote Sensing 44 (2) (2006) 397-408.

[9] S.-L. Chen, X.-Y. Hu, S.-L. Peng, Hyperspectral imagery denoising using a spatial-spectral domain mixing prior, Journal of computer science and technology 27 (4) (2012) 851-861.

[10] E. J. Candès, X. Li, Y. Ma, J. Wright, Robust principal component analysis?, Journal of the ACM (JACM) 58 (3) (2011) 11.

[11] E. Candes, B. Recht, Exact matrix completion via convex optimization, Communications of the ACM 55 (6) (2012) 111-119.

[12] I. Jolliffe, Principal component analysis, Wiley Online Library, 2002.

[13] R. Cabral, F. De la Torre, J. P. Costeira, A. Bernardino, Unifying nuclear nor$\mathrm{m}$ and bilinear factorization approaches for low-rank matrix decomposition, in: Proceedings of the IEEE International Conference on Computer Vision, 2013, pp. 2488-2495.

[14] Y. Shen, Z. Wen, Y. Zhang, Augmented lagrangian alternating direction method for matrix separation based on low-rank factorization, Optimization Methods and Software 29 (2) (2014) 239-263.

[15] H. Ji, C. Liu, Z. Shen, Y. Xu, Robust video denoising using low rank matrix completion, in: Computer Vision and Pattern Recognition (CVPR), 2010 IEEE Conference on, IEEE, 2010, pp. 1791-1798.

[16] C. A. Bishop, J. G. Liu, P. J. Mason, Hyperspectral remote sensing for mineral exploration in pulang, yunnan province, china, International Journal of Remote Sensing 32 (9) (2011) 2409-2426. 
[17] L. Wu, A. Ganesh, B. Shi, Y. Matsushita, Y. Wang, Y. Ma, Robust photometric stereo via low-rank matrix completion and recovery, Computer Vision-ACCV 2010 (2011) 703-717.

[18] B. Fischler, Martin A, R. C, Random sample consensus: a paradigm for model fitting with applications to image analysis and automated cartography, Communications of the ACM 24 (6) (1981) 381-395.

[19] Y.-Q. Zhao, P. Gong, Q. Pan, Object detection by spectropolarimeteric imagery fusion, IEEE Transactions on Geoscience and Remote Sensing 46 (10) (2008) 3337-3345.

[20] F. De La Torre, M. J. Black, A framework for robust subspace learning, International Journal of Computer Vision 54 (1) (2003) 117-142.

[21] Y. Q. Zhao, J. Yang, Hyperspectral image denoising via sparse representation and low-rank constraint, IEEE Transactions on Geoscience and Remote Sensing 53 (1) (2015) 296-308.

[22] H. Huang, A. G. Christodoulou, W. Sun, Super-resolution hyperspectral imaging with unknown blurring by low-rank and group-sparse modeling, in: IEEE International Conference on Image Processing, 2014, pp. 2155-2159.

[23] X. Lu, Y. Wang, Y. Yuan, Graph-regularized low-rank representation for destriping of hyperspectral images, IEEE Transactions on Geoscience and Remote Sensing 51 (7) (2013) 4009-4018.

[24] H. Zhang, W. He, L. Zhang, H. Shen, Q. Yuan, Hyperspectral image restoration using low-rank matrix recovery, IEEE Transactions on Geoscience and Remote Sensing 52 (8) (2014) 4729-4743.

[25] T.-H. Oh, Y.-W. Tai, J.-C. Bazin, H. Kim, I. S. Kweon, Partial sum minimization of singular values in robust pca: Algorithm and applications, IEEE transactions on pattern analysis and machine intelligence 38 (4) (2016) 744-758. 
[26] Z. Lin, M. Chen, Y. Ma, The augmented lagrange multiplier method for exact recovery of corrupted low-rank matrices, arXiv preprint arXiv:1009.5055.

[27] D. L. Donoho, I. M. Johnstone, Adapting to unknown smoothness via wavelet shrinkage, Journal of the american statistical association 90 (432) (1995) 12001224.

[28] E. T. Hale, W. Yin, Y. Zhang, Fixed-point continuation for $L_{1}$-minimization: Methodology and convergence, SIAM Journal on Optimization 19 (3) (2008) $1107-1130$.

[29] E. M. de Sá, Exposed faces and duality for symmetric and unitarily invariant norms, Linear Algebra and its Applications 197 (1994) 429-450.

[30] N. Shahid, V. Kalofolias, X. Bresson, M. Bronstein, P. Vandergheynst, Robust principal component analysis on graphs, in: Proceedings of the IEEE International Conference on Computer Vision, 2015, pp. 2812-2820.

[31] J. Ma, C. Li, Y. Ma, Z. Wang, Hyperspectral image denoising based on low-rank representation and superpixel segmentation, in: Image Processing (ICIP), 2016 IEEE International Conference on, IEEE, 2016, pp. 3086-3090.

[32] D. Landgrebe, Aviris nw indianas indian pines 1992 data set, 1992.

[33] A. Plaza, J. A. Benediktsson, J. W. Boardman, J. Brazile, L. Bruzzone, G. CampsValls, J. Chanussot, M. Fauvel, P. Gamba, A. Gualtieri, et al., Recent advances in techniques for hyperspectral image processing, Remote sensing of environment 113 (2009) S110-S122.

[34] A. L. Neuenschwander, Remote sensing of vegetation dynamics in response to flooding and fire in the Okavango Delta, Botswana, The University of Texas at Austin, 2007. 